\section{Cognitive Perspective}

Edward Nęcka

Institute of Psychology, Jagiellonian University, Kraków, Poland

\section{Definition}

Cognitive perspective in the study of individual differences amounts to analyzing human traits in terms of underlying cognitive processes. The way in which we perceive the world, attend to the external stimuli, store information in short-term and long-term memory, or solve problems via the process of thinking defines what we are like. The cognitive perspective allows integration of the psychology of individual differences with the experimental cognitive psychology.

\section{General Issues}

Cognitive analysis of individual differences is a relatively new approach in psychological science. Contemporary cognitive psychology evolved in the $1950 \mathrm{~s}$ and $1960 \mathrm{~s}$ as a continuation of the traditional experimental psychology, whereas the psychology of individual differences developed from the so-called correlational psychology (Cronbach 1957), also known as the differential psychology. These two disciplines of scientific psychology used to differ in basic theoretical and methodological assumptions. Experimental psychologists focused on the general rules of behavior, thus ignoring the fact that one can hardly find two identically behaving animal subjects or human participants. In typical experimental settings, participants were expected to behave in the specific manner due to deliberate experimental manipulations, not because of their personal traits. Therefore, individual differences were treated as a source of unwanted variance that should be reduced, if possible, because the greater such a variance, the lower the chances to reject the null hypothesis. Consequently, experimental psychologists were interested in making the groups of participants as homogeneous as possible in reference to their temperament, personality, intelligence, diligence, and other personal traits. In contrast, differential psychologists were primarily interested in between-person variability of traits; therefore, they sought for the most differentiated samples possible to find. Since differential psychologist relied mostly on the correlational approach, their preference for large and diversified samples was absolutely logical because large amount of within-sample variance usually increases the probability of finding significant correlations between various aspects of human individuality. Restricted variance, on the other hand, usually makes the investigated relationships statistically not significant. So, for the experimental psychologists, the phenomenon of individual differences was somewhat uncomfortable as a "noise," whereas for the differential psychologist, 
who usually did not carry out controlled laboratory experiments, this phenomenon lied just in the focus of their scientific interest.

Experimental and differential psychology have been developing fairly in isolation until the 1970s, when the pioneering studies by Earl Hunt (Hunt et al. 1973; Hunt 1980) and Robert J. Sternberg (1977) initiated fruitful combination of these two perspectives. For instance, Earl Hunt and his coworkers discovered that people of high verbal ability, in comparison to less endowed persons, were quicker in the basic cognitive operation of letter comparison. An idea thus emerged that complex intellectual abilities, investigated by differential psychologists, might result from the speed of elementary cognitive operations, the latter being investigated with the purely "nondifferential" paradigms developed by cognitive psychologists. Robert J. Sternberg, on the other hand, developed so-called componential analysis that allowed splitting the general mental ability, measured with IQ tests, into separate cognitive building blocks (i.e., components). This approach allowed definition of general intelligence in terms of the cognitive process and its constituents rather than in terms of a bit mythical entity comprising of latent "factors." In this way, Sternberg demonstrated that the structural approach, typical of differential psychology, may be, and should be, accompanied by the processual approach, typical of experimental cognitive psychology.

Today, the combination of experimental and differential perspectives is a standard approach in the study of individual differences, as well as in the study of general cognition. Such a combination allows deeper insight into the nature of both human individuality and human cognition. Specifically, this approach consists in investigation of the following questions:

1. What are the cognitive correlates of various dimensions of human individuality? Thanks to this kind of research, psychologists accumulate knowledge about cognitive aspects of such traits as personality, intelligence, creativity, or self-control.

2. If such a relationship is found, which factors determine its strength? Precisely, such a relationship may rely on specific components, or stages, of the cognitive process. For instance, Sternberg found that general intelligence is related mostly to one specific component of the process of analogical thinking, namely, the operation of mapping one cognitive structure against another one. Consequently, the author suggested that speed and accuracy of mapping might account for the general factor of intelligence, whereas speed or accuracy of other components did not count very much. Alternatively, such a relationship may appear only in specific experimental conditions, characterized by increased cognitive demands. For instance, Hunt and Lansman found that high-ability people did not differ from low-ability ones in the single-task conditions but started to excel as soon as task conditions became much more demanding, as is the case of dual-task performance. Such findings are particularly interesting because they may suggest possible theoretical explanation of the nature of the trait versus process relationships.

3. If such a relationship is found, what does it mean in terms of the direction of causation? This question is the most difficult one because correlational studies, including the structural equation modeling (SEM) approach, do not allow causal explanations. The latter usually needs experimental manipulation with independent variables. For instance, a relationship between intelligence and dual-task performance may suggest that (1) the multitasking ability is determined by the general mental ability, (2) intelligence is explained by the multitasking ability as one of its underlying determinants, or (3) there is a third element (e.g., a neural or genetic factor) which determines both variables found to be correlated. Since we cannot manipulate with the level of intelligence, we must rely on indirect inference or theoretical considerations. Fortunately, the cognitive approach suggests promising ways to get insight into the causal nature of many correlational relationships. This is because the strength of a relationship between two variables may vary if one of them is 
experimentally manipulated in reference to specific cognitive requirements. Such an approach is sometimes called a "quasiexperimental approach" (Hambrick et al. 2005), and it consists in treating the correlation between a measure of an elementary cognitive function and a measure of a higher-order trait as a dependent variable, whose value may fluctuate according to the experimental manipulations with cognitive load.

Thus, the cognitive approach to individual differences allows deeper understanding not only how people differ one from another but also why do they differ and what are the underlying factors of important individual traits. For these reasons, the cognitive perspective seems quite promising from the methodological and theoretical point of view.

\section{Intelligence}

The cognitive approach to intelligence started with reaction time studies, which showed that IQ was negatively correlated with both simple and choice reaction time (Jensen 1998). Though reaction time, as well as inspection time that also used to be studied in this context (Deary 1994), is not a cognitive process per se, it is believed to reflect an important characteristic of cognition, namely, the processing speed. Therefore, these findings corroborated the hypothesis according to which intelligence may be explained by speed of mental processing, in other words, by the velocity of elementary cognitive processes.

Today, the mainstream of research on cognitive correlates of intelligence pertains to working memory capacity (WMC), defined as the ability to memorize the incoming data for a short time and to operate on them according to the current task's requirements. Models of working memory emphasize its dual nature as both "a memory" and a mental mechanism responsible for information processing (Baddeley 1986). Two issues are important while theorizing about the significance of WMC for human intellectual functioning. Firstly, working memory is severely limited in its capacity, defined in terms of both the amount of information possible to be maintained for a short period of time and the duration of such maintenance. Secondly, there are vast individual differences in WMC that can be assessed with dedicated tests, such as the OSPAN procedure. In other words, people substantially differ in their WMC, which - by the way - is subjected to hard limitations. Hence, the question arises whether individual differences in WMC correlate with individual differences in other aspects of human cognition, such as language processing, problem solving, and general fluid intelligence (Gf). As to the relationships between WMC and Gf, various studies demonstrated that these measures share from $50 \%$ to $70 \%$ of common variance (Chuderski and Nęcka 2012). It means that the cognitive processes involved in working memory functioning explain the substantial part of the general intellectual ability. It also implies that $\mathrm{WMC}$ is probably the strongest known predictor of Gf.

Having established the importance of WMC for the general fluid intelligence, researches started to ask which aspects of working memory are responsible for this link. Two answers may be found in the literature. According to the first stance, it is the storage capacity of WM that decides about the quality of abstract reasoning and - consequently - the level of intelligence. Storage capacity is not synonymous with working memory capacity (WMC); rather, it is just one of its determinants. Storage capacity may be defined in terms of the number of chunks of information possible to be stored and actively maintained for a short time, whereas WMC is a term pertaining to the general efficiency of the working memory mechanism. Apart from storage capacity, WMC is determined by speed and efficacy of specific operations performed by WM, such as substitution of elements, inhibition of intrusions, or speed of removal of elements that are not relevant anymore. If general fluid intelligence is determined mostly by storage capacity, it may be predicted on the basis of the so-called span tests that allow assessment of the number of elements possible to hold in memory for a short time. 
According to the second stance, general fluid intelligence depends on the function of controlled attention. For instance, Nelson Cowan (1995) claims that working memory is just the active part of long-term memory. Activated chunks of information are ready for use in current processing, whereas less active ones have to be activated first. Controlled attention is one of the mechanisms that subserve such activation. Therefore, the efficiency of cognitive control determines the overall capacity of working memory. Consequently, it also determines the general level of intelligence. Randall Engle and his team (Engle et al. 1999) share this stance, claiming that efficacy of cognitive control, particularly efficacy of controlled attention, is the most important determinant of Gf.

Standard working memory tests do not allow clear conclusion concerning these two competing accounts of the nature of Gf/WMC relationship. For instance, the OSPAN test that is usually employed in these studies consists in simultaneous performance of two tasks. The first task needs verification of series of the arithmetic equations, and the second one needs remembering words that appear at the end of every equation formula. In such a way, OSPAN engages two theoretically relevant aspects of working memory, namely, storage and processing. However, it does not allow splitting these two aspects at the measurement level in order to verify the competing hypotheses about the Gf/WMC relationship. In order to do so, researchers have to employ two independent batteries of WM tasks, one pertaining mostly to storage capacity and another one relating mainly to executive control. Another solution amounts to employment of one task that provides independent markers of storage capacity and executive control. For instance, a special version of n-back task meets these requirements. In this task, a series of stimuli (e.g., two-digit numbers) appear and disappear at the screen one by one. The task is to recognize repetition of any stimulus that has been already presented $n$ items back. Manipulation with the number $n$ makes the task more or less difficult, since recognition is easy in the case of immediate repetition (e.g., 56, 56, $n=1)$ and it gets quite difficult if it is mediated by other elements (e.g., 56, 78, 42, 56, $n=3$ ). Additionally, the sequence of stimuli may involve lures, that is, stimuli that are repeated too early or too late and thus should be ignored. For instance, if the participants are instructed that $n=2$, the repetitions on the position $n=1$ or $n=3$ are just irrelevant lures, although they may prompt impulsive erroneous responses. In such a research paradigm, the number of correct responses to targets (e.g., $n=2$ ) is a measure of storage capacity, whereas the number of correct rejection of lures (e.g., $n=1$ or $n=3$ ) is a measure of the efficacy of inhibitory control. Adopting such a methodology, we (Chuderski and Nęcka 2012) were able to establish that storage capacity is a stronger predictor of Gf than executive control. However, impurity of tasks used in cognitive experiments, including the n-back task, prompted us to adopt still another approach, in which we used two batteries of various tasks pertaining more to storage capacity than executive control, or vice versa. Using the structural equation modeling (SEM), we were able to demonstrate that the latent variable pertaining to executive control has very limited explanatory power concerning Gf, whereas storage capacity explains almost the entire pool of Gf variance (Chuderski et al. 2012).

It is still far from clear which aspects of working memory predict general fluid intelligence. According to the relational integration hypothesis, intelligence depends on the ability to create temporary bindings between mental representations in order to obtain more complex relational structures (Oberauer et al. 2007). The process of binding is believed to be crucial for abstract reasoning, because it allows speedy combination and recombination of premises that allow suitable conclusion. What counts in relational integration is both the number of representations possible to bind in memory for a short time and the speed with which old bindings are replaced with new ones. Efficiency of these processes is claimed to be the best predictor of the quality of abstract reasoning (Chuderski 2014), also known as general fluid intelligence. 


\section{Self-Control}

Self-control is an ability to define and pursue long-distance goals in spite of situational influences, external pressures, or current physiological needs of the organism. It is an important predictor of life success, including health, wealth, and social status. Its failures may be observed when somebody loses control over his/her own behavior and yields to the current impulses, usually at the expense of future gains.

Self-control may be conceptualized as both a process and a trait. As a process, it consists of several stages playing various roles in selfregulation of behavior (see Kotabe and Hofmann 2015). First of all, a person must realize that there is a conflict between one of his/her long-distance goals and a current desire or temptation. For instance, the goal to obtain university degree, which requires years of hard work and effort, may be difficult to bring together with the need to socialize or with a temptation to prolong vacationing. People usually strive for many goals of different importance and urgency, so they must learn how to combine them with their limited resources, such as time and energy necessary to exert sustained control over their behavior. But primarily they must be able to detect conflict between the goals, particularly between important long-distance objectives and transient desires. The next stage consists in mobilization of resources necessary to exert control. Acts of selfcontrol are costly in terms of energy and effort, meaning that people are less likely to control themselves after having completed demanding tasks. The so-called "ego depletion" effect (Baumeister et al. 2007) has been demonstrated in the sequence task paradigm, where people are asked to do a task that requires controlled attention (e.g., the Stroop task) after having done another task of similar nature (e.g., trying not to read subtitles while watching a movie). Although the "ego depletion" effect (Baumeister et al. 1998) is hotly debated concerning its strength and underlying mechanisms, it has been convincingly demonstrated that acts of self-control deprive us a portion of our cognitive and/or energetic resources needed either to continue this very act of self-control or to start a new effortful task. Hence, detection of the conflict between a longdistance goal and a current desire must be followed by mobilization or restoration of energetic as well as cognitive resources; otherwise the process of self-control is likely to fail. The third stage consists in enactment of effective motivation to exert self-control. It happens that a person is able to detect the goal vs. desire conflict, as well as he/she has enough resources available, and still self-control is not exerted due to lack of the decision to do so. In other words, an act of self-control must be consciously decided upon if it is to take place at all, the more so that there are usually many competing goals of equal or even higher attractiveness. Finally, a person who decided to exert self-control must flexibly adjust to the everchanging conditions of the environment. It means that he/she has to (1) maintain the important longdistance goal in working memory; (2) inhibit unwanted reactions, impulses, thoughts, or feelings; and (3) switch from one action to another according to requirements of the current situation.

As a trait, self-control is a relatively stable disposition with a considerable predictive power concerning various measures of life success (Casey et al. 2011). It may be conceptualized as the individual ability to perform properly and effectively the abovementioned stages of the process of self-control. There are individual differences in the ability to detect conflict between long-distance goals and current desires, as well as in the ability to mobilize resources, to motivate oneself for the exertion of self-control, and to flexibly adjust one's behavior to the requirements caused by the current situation. In other words, every stage of the process of self-control may succeed or fail, and the (low) probability of failures implies (high) level of the trait of self-control. This dimension of individual differences is stable enough to serve as a correlate of income, professional achievement, and life satisfaction.

The trait of self-control is measured in two ways. The first group of assessment tools includes questionnaires, such as the Self-Control Scale (Tangney et al. 2004) or the NAS-50 scale (Nęcka et al. 2016). These questionnaires consist of items referring to various real-life situations in 
which one's self-control may succeed or fail. For instance, a person is asked about his/her ability to maintain goals, adaptively switch from one action to another, inhibit unwanted actions, or do deadlines. Although the self-control questionnaires show good psychometric characteristics concerning reliability and validity, they are criticized for relying on people's self-awareness, which may be far from perfect as far as the control abilities are concerned. For these reasons, scales of self-control are sometimes constructed in both the first-person and the third-person versions (e.g., Nęcka et al. 2016), the latter allowing assessment provided by informants (i.e., family members, coworkers, colleagues). The second group of tools consists of standardized observational scales (e.g., Moffitt et al. 2011), thanks to which informants (e.g., parents or teachers) can provide information about the participant's level of self-control exerted in real-life situations. The observational scales do not rely on self-reporting, so they are regarded more valuable assessment tools in comparison to typical questionnaires. However, such scales are suitable only for careful and attentive informants, who are not easily found in typical research paradigms.

Cognitive underpinnings of trait self-control are called executive functions (EF). These higher-order processes are responsible for adaptive control and coordination of cognition in the service of goal-directed activity. According to the popular taxonomy by Akira Miyake and his coworkers (Miyake et al. 2000), there are three major EFs: inhibition, shifting, and working memory updating. The first function is responsible for prepotent response inhibition when such a response would be harmful or at least inappropriate. Suppression of one's inclination to offend another person may be a good example of efficient inhibitory control. The second function, shifting, is responsible for adaptive switching between different tasks, types of activity, or mindsets. Inefficient shifting results either in perseverance instead of switching into another action or at least in the increased costs of switching (i.e., longer response time or higher probability of error). People who tend to repeat the same joke in every occasion seem to suffer from inefficient shifting.
The third function is working memory updating, thanks to which people are aware of the current situation and its cognitive requirements. People who do not update their working memory store quickly and properly tend to respond to irrelevant cues or are lost in their inner mental states, thus neglecting the demands caused by current tasks.

The list of executive functions proposed by Miyake is probably not complete. First of all, it lacks the function of planning, without which acts of self-control cannot be exerted. Next, the function of dual- or multitasking is worth consideration. Efficient self-control often requires that more than one action is necessary to perform, and people vary extensively in terms the multitasking abilities. Another problem connected with the Miyake model pertains to "unity and diversity" of executive functions, which amounts to the question of their mutual intercorrelations. It appears the function of shifting is the least connected with others, whereas the function of inhibition tends to be the most general. Even though EFs are interrelated, they are still specific enough to account for very special aspects of selfcontrol, which means that the trait of self-control should be investigated and assessed according to multidimensional models consisting of several subscales rather than one general factor (see Nęcka et al. 2016).

The major problem concerning the cognitive underpinnings of self-control amounts to the fact that those executive functions pertain mostly to the third stage of the process of self-control. This final step of flexible implementation of behavioral plans is probably the best investigated from the cognitive point of view, because we can argue that working memory updating is responsible for goal maintenance, inhibitory control (for abstaining from unwanted or improper actions), and task switching (for adaptive adjustment of behavior). However, there is not enough knowledge concerning the cognitive aspects of other stages of the process of self-control, such as conflict detection, setting up effective motivation, and mobilization of resources. 


\section{Personality}

One of the basic issues discussed in the field of the psychology of personality is the trait versus situation problem. For traditional differential psychologists, these are stable dispositions (i.e., traits) that determine human behavior and make it relatively invariant across situations. According to such an explanation, an aggressive person will demonstrate increased probability of violent behavior regardless of current situational factors, although the situation may moderate the likelihood of aggression; it may also influence the way in which aggressive acts would occur. This line of theorizing is particularly popular among the differential psychologists who search for genetic predispositions of personality traits. For the proponents of situational explanations, it is the current situation that influences human behavior most, particularly the social cues. These theorists maintain that the same person may behave in the aggressive or peaceful way depending on such factors as the presence or absence of specific triggers of aggression or the way other people behave at the same time.

The trait versus situation debate has been addressed by Walter Mischel and Shoda (1995) who developed the so-called cognitive-affective model of personality. The authors do not ignore the fact that human behavior is consistent across different situations; however, they ascribe much more significance to the consistency within situations. For instance, aggression is likely to appear again and again in an individual life history only if the characteristics of situations are repeated, too. In another situation the same person may respond in the peaceful and cooperative way, thus suggesting that he/she lacks any "disposition to aggression" or maybe he/she has the "disposition to agreeableness." Instead of relying in dispositional accounts, Mischel and Shoda underscore the importance of the "if-then" patterns that combine situational factors (i.e., if something happens) with specific behavioral outcomes (i.e., then a given response is produced). The "ifthen" patterns are formed and stored in the cognitive-affective personality system (CAPS), thanks to which human behavior is both dependent on the situation and constant over time.
The CAPS system involves five units, namely, (1) encoding, storage, and use of information, (2) beliefs and expectations concerning the outcomes of one's actions, (3) goals and values that determine one's motivation, (4) affective patterns of responding in a given situation, and (5) competencies, such as knowledge and abilities. Personality is defined as a unique pattern of these units, thanks to which effective regulation of behavior is possible.

Michel and Shoda's model of personality is a good example of the cognitive approach. According to this stance, human personality is determined by the way in which we perceive and understand the world, other people, and ourselves as well. The classic example of such an approach is George Kelly's (1995) theory of personal constructs. The author assumes that people are naïve scientists, meaning that they construct and verify hypotheses about the world, especially the social world. The personally verified hypotheses are becoming parts of the system of personal constructs, which allow understanding what is going on in the environment and predicting what is likely to happen next. Such personal constructs are relatively constant over time, for two reasons. First, they are the result of hard work and effort, so they tend to be defended as long as new evidence suggests their rejection or modification. Second, they are parts of the complex system, so any change of a given construct may be consequential for other constructs. Therefore, human behavior regulated by the system of personal constructs is relatively stable across situations. It is also highly idiosyncratic, that is, typical for a given person who usually differs from other people in the way he/she behaves in the given circumstances. The theory of personal constructs is a perfect example of the cognitive approach because it underscores the role of cognition both in the regulation of behavior and in the process of personality development, the latter being just the process of building the system of personal construct through verification of hypotheses.

Apart from developing the cognitive models of personality as a system of behavior regulation that relies on the way people perceive and understand the world, the cognitive perspective may lead to quite another research agenda. This research area 
is involved in studying how various personality traits affect cognitive functioning. Personality dimensions assessed with standard psychometric tools are treated as possible moderators of general rules of cognition, investigated within the experimental paradigm. Within such an approach, Błażej Szymura (2010) found that three superfactors of personality conceptualized by Hans J. Eysenck $(1967,1981)$ influence the functioning of attention, although the nature of such influence depends on the particular dimension. The dimension of extraversion/introversion appeared important for dual tasking, since extraverts tended to outperform introverts when the task conditions required that two tasks be performed simultaneously. Neuroticism appeared relevant for the attentional function of selectivity because the data suggested that highly neurotic persons committed performed worse than the emotionally stable people when task conditions required rapid selection of relevant items and ignoring irrelevant ones. When the speed of presentation was not increased, neuroticism did not predict performance in the attention test. Finally, the trait of psychoticism moderated performance in the sustained attention test, suggesting that people characterized by high level of the P trait suffer from inability to sustain attention over time. In other studies, the $\mathrm{P}$ trait also showed significant relationships with leaks of attention, that is, the impaired ability to screen out currently irrelevant stimuli.

Studies showing that personality traits are apt to moderate the outcomes of cognitive processes, and maybe their progression as well, are important for several reasons. Firstly, they happen to be relevant for the theoretical debates concerning the nature and structure of personality. For instance, Szymura (2010) claims that his research findings support the biological model of personality suggested by H. J. Eysenck. Secondly, these findings suggest that general rules of cognition are not that general as they are sometimes believed to be. Individual differences should not be treated as a "noise" contaminating the results of "purely" cognitive experiments. Finally, such findings may have practical implications because they suggest that people characterized by some personality dispositions should not be recruited for jobs characterized by specific cognitive requirements. The studies on personality correlates of attention seem particularly important form this point of view.

\section{Conclusion}

The science of individual differences emerged as an attempt to describe and understand the phenomenon of interindividual differentiation of human behavior. Between-person differences in responding to external stimuli had to be accounted for in terms of underlying factors. Broadly speaking, there are three modes of explanation adopted by the scholars of individual differences, corresponding to three levels of theorizing. The first one amounts to looking for genetic determination of individual traits. The second one consists in linking individual traits with pertinent neural mechanisms. The third one relies on construction of cognitive models of human personal traits. Thus, the cognitive perspective is just one of a few possible ways to grasp the problem of interindividual differentiation of human behavior. All perspectives should be taken together in order to obtain deeper understanding of intelligence, self-control, and personality.

Acknowledgments Preparation of this contribution was supported by the Polish National Centre of Science (NCN), grant No. DEC-2013/08/A/HS6/00045.

\section{References}

Baddeley, A. (1986). Working memory. Oxford: Clarendon Press.

Baumeister, R. F., Bratslavsky, E., Muraven, M., \& Tice, D. M. (1998). Ego depletion: Is the active self a limited resource? Journal of Personality and Social Psychology, 74, 1252-1265.

Baumeister, R. F., Vohs, K. D., \& Tice, D. M. (2007). The strength model of self-control. Current Directions in Psychological Science, 16(6), 351-355.

Casey, B. J., Somerville, L. H., Gotlib, I. H., Ayduk, O., Franklin, N. T., Askren, M. K., Jonides, J., Berman, M. G., Wilson, N. L., Teslovich, T., Glover, G., Zayas, V., Mischel, W., \& Shoda, Y. (2011). Behavioral and neural correlates of delay of 
gratification 40 years later. Proceedings of the National Academy of Sciences USA, 108(36), 14998-15003.

Chuderski, A. (2014). The relational integration task explains fluid reasoning above and beyond other working memory tasks. Memory and Cognition, 42(3), 448-463.

Chuderski, A., \& Nęcka, E. (2012). The contribution of working memory to fluid reasoning: Capacity, control, or both? Journal of Experimental Psychology: Learning, Memory, and Cognition, 38(6), 1689-1710.

Chuderski, A., Taraday, M., Nęcka, E., \& Smoleń, T. (2012). Storage capacity explains fluid intelligence but executive control does not. Intelligence, 40(3), 278-295.

Cowan, N. (1995). Attention and memory: An integrated framework. New York: Oxford University Press.

Cronbach, L. J. (1957). The two disciplines of scientific psychology. American Psychologist, 12, 671-684.

Deary, I. J. (1994). Sensory discrimination and intelligence: Postmortem or resurrection? American Journal of Psychology, 107, 95-115.

Engle, R. W., Tuholski, S. W., Laughlin, J. E., \& Conway, A. R. A. (1999). Working memory, shortterm memory, and general fluid intelligence: A latent variable approach. Journal of Experimental Psychology: General, 128, 309-331.

Eysenck, H. J. (1967). The biological basis of personality. Springfield: Thomas.

Eysenck, H. J. (1981). General features of the model. In H. J. Eysenck (Ed.), A model of personality (pp. 1-37). Berlin: Springer.

Hambrick, D. Z., Engle, R. W., \& Kane, M. J. (2005). The role of working memory in higher-level cognition: Domain-specific vs. domain-general perspectives. In R. J. Sternberg \& J. Pretz (Eds.), Intelligence and cognition (pp. 104-121). New York: Cambridge University Press.

Hunt, E. B. (1980). Intelligence as an information processing concept. The British Journal of Psychology, 71, 449-474.

Hunt, E., Frost, N., \& Lunneborg, C. (1973). Individual differences in cognition: A new approach to intelligence. Psychology of Learning and Motivation, 7, 87-122.

Jensen, A. R. (1998). The g factor: The science of mental ability. Westport: Praeger.
Kelly, G. (1995). The psychology of personal constructs. New York: W. W. Norton.

Kotabe, H. P., \& Hofmann, W. (2015). On integrating the components of self-control. Perspectives on Psychological Science, 10(5), 618-638.

Mischel, W., \& Shoda, Y. (1995). A cognitive-affective system theory of personality: Reconceptualizing situations, dispositions, dynamics, and invariance in personality structure. Psychological Review, 102(2), 246-268. https://doi.org/10.1037/0033-295X.102.2.246.

Miyake, A., Friedman, N. P., Emerson, M. J., Witzki, A. H., \& Howerter, A. (2000). The unity and diversity of executive functions and their contributions to complex 'frontal lobe' tasks: A latent variable analysis. Cognitive Psychology, 41, 49-100.

Moffitt, T. E., Arseneault, L., Belsky, D., Dickson, N., Hancox, R. J., Harrington, H., Houts, R., Poulton, R., Roberts, B. W., Ross, S., Sears, M. R., Thomson, W. M., \& Caspi, A. (2011). A gradient of childhood self-control predicts health, wealth, and public safety. Proceedings of the National Academy of Sciences USA, 108, 2693-2698.

Nęcka, E., Wujcik, R., Orzechowski, J., Gruszka, A., Janik, B. Nowak, M., \& Wójcik, N. (2016). NAS-50 and NAS-40: New scales for the assessment of selfcontrol. Polish Psychological Bulletin, 47(3), 346-355. https://doi.org/10.1515/ppb-20016-0041.

Oberauer, K., Süß, H.-M., Wilhelm, O., \& Sander, N. (2007). Individual differences in working memory capacity and reasoning ability. In A. R. A. Conway, C. Jarrold, M. J. Kane, A. Miyake, \& J. N. Towse (Eds.), Variation in working memory (pp. 49-75). Oxford: Oxford University Press.

Sternberg, R. J. (1977). Intelligence, information processing, and analogical reasoning: The componential analysis of human abilities. Hillsdale: Erlbaum.

Szymura, B. (2010). Individual differences in resource allocation policy. In A. Gruszka, G. Matthews, \& B. Szymura (Eds.), Handbook of individual differences in cognition: Attention, memory, and executive control (pp. 231-246). New York: Springer.

Tangney, J. P., Baumeister, R. F., \& Boone, A. L. (2004). High self-control predicts good adjustment, less pathology, better grades, and interpersonal success. Journal of Personality, 72, 271-322. 